\title{
WIP: Examining Micro-interventions to Improve Classroom Community in Introductory Engineering Classrooms
}

\section{Ms. Christine Allison Gray, Northern Arizona University}

Christine Allison Gray is a doctoral student in the College of Education at Northern Arizona University. She also serves as a graduate assistant on the Reshaping Norms project in the College of Engineering, Forestry and Natural Sciences. Her research focuses on the influence of classroom climate on the development of undergraduate students' professional engineering identity.

\section{Dr. Robin G. Tuchscherer, Northern Arizona University}

Dr. Tuchscherer currently serves as an Assistant Professor at Northern Arizona University where he has taught since 2011. Prior to academia, he accumulated eight years of professional experience as a practicing structural engineer and brings a practitioner's perspective to the academic and research setting. He teaches core undergraduate engineering courses, structural analysis, and reinforced concrete design. His primary research focus is related to improving our understanding of the design and behavior of concrete structures; and he is actively involved within the professional engineering community. Furthermore, Dr. Tuchscherer has also supervised sponsored research and educational reform initiatives related to the improvement of student learning.

\section{Dr. Ron Gray, Northern Arizona University}

Ron Gray, Ph.D. is an assistant professor of science education in the Center for Science Teaching and Learning at Northern Arizona University. He graduated from Oregon State University with a doctorate in science education. His work largely focuses on providing secondary science teachers the tools to design and implement learning experiences for their students that are effective and authentic to the discipline. Much of this work has been centered on model-based inquiry and the integration of scientific practices in a supportive and structured way. He has been funded by NSF and other agencies to conduct research on preservice teacher education, undergraduate engineering education, and community partnerships in secondary education. 


\section{WIP: Examining micro-interventions to improve classroom community in introductory engineering classrooms}

The field of engineering education, like many areas in higher education, is steeped in tradition. Engineering departments are known for traditional lecture-style classrooms with high enrollment, particularly at the lower levels, where direct instruction, along with grades based largely on a handful of multiple choice exams, are the norm [1]. Introductory courses -- the start of an unforgiving workload -- serve to "weed out" students at an early stage, and typically result in large numbers of D, F and W grades, which often leads students to drop the major [2]. The curriculum in these lower-level courses typically focuses on solving abstract problems that meld mathematics and physics, but may not stretch into an area that students recognize as "engineering" [3]. Research shows that these factors influence student persistence and attitudes about the field [1]. Engineering students have an attrition rate near 50 percent, with minority students dropping out of the engineering degree at higher rates than white students [4]. In addition, engineering remains a STEM field still unable to achieve gender parity [5].

Although academic rigor is often blamed for this "leaky pipeline," the culture and tradition within engineering classrooms may be a more significant factor [6]. Many educators are content experts, but may have minimal training in translating that content into curriculum for students; engineering professors may feel unable or unwilling to make large-scale changes to the existing curriculum, despite research-based recognition that many of the traditions in STEM fields contribute to student attrition, and to the lack of diversity and gender parity in the field [1]. Thus, there is a need to create interventions on a "micro" scale that may be easily integrated into the already-substantial engineering curriculum. These small-scale, easy-to-implement changes may be more easily accessible by engineering faculty.

In this study, we implement a suite of "micro-interventions," or small actions that instructors can implement to create significant effects in the ways that students view, and feel about, engineering. The micro-interventions are focused on increasing the students' sense of community, defined as a feeling of "spirit, trust, mutual interdependence among members, interactivity, shared valued and beliefs, and common expectations" [7].

The micro-interventions alter the manner in which students interact with each other, with the instructor, and with the curriculum. Central to the interventions are classroom norms (see Figure 1) which are introduced on the first day and explicitly referenced throughout the term. The specific norms are meant to increase equitable student talk and interactions in the classroom while highlighting reasoning over correct answers. The micro-interventions in this study include: 1) explicit attention to norms in the classroom and in engineering; 2) strategies to increase peer-to-peer interactions; 3 ) strategies to increase rapport between the instructor and students; 4) peer testimonials to enable discussions of the challenges faced by first-year engineering students, and 5) interactive learning strategies. Each micro-intervention was developed in collaboration with the grant research team, and is being designed and tested over two years in three separate iterations, with a

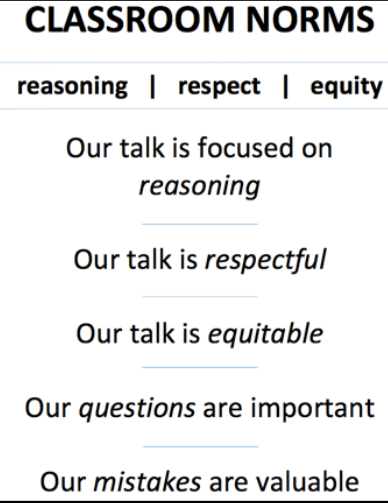

Figure 1. Classroom norms poster 
final pilot study occurring in Spring 2018. This paper focuses on the baseline data and describes the ongoing interventions and anticipated results in the first iteration. The research question guiding this work is: To what extent do the micro-interventions affect the sense of classroom community of an introductory engineering course?

\section{Setting and participants}

Data collection is taking place in and around two separate sections of a 200 -level engineering course ("Applied Mechanics Statics") taught in the engineering department at a large public university in the southwest United States. One section serves as the treatment and the second as the control. The treatment section began with an enrollment of 62 students, and the control section with an enrollment of 92 students. This course was chosen because of its status as a gatekeeper course within engineering, and because of its departmental history as a course with high numbers of $\mathrm{D}, \mathrm{F}$, and $\mathrm{W}$ grades. The course is required by all civil, mechanical, and environmental engineering majors. The content of Statics provides students with their first taste of a technical engineering context, applying concepts learned in advanced mathematics and physics to engineering problems; however, the course has traditionally presented problems in an abstract, textbook-based style. Statics is widely considered by students and faculty as a "weedout" course: a traditionally rigorous class with high rates of low grades and failures that drives large numbers of students out of the major [8]. Female and minority students, who number significantly fewer than other students at the start of the course, become an even smaller group of individuals who are able to move on in the engineering major after the course is over.

\section{Data collection and analysis}

The study follows a quasi-experimental, multi-method design to answer the research question. We utilize two quantitative measures along with semi-structured interviews of a sub-sample of participants in order to triangulate the results of the quantitative measures. The two quantitative measures consist of an observational protocol to measure instruction and student participation, and a survey instrument designed to measure students' sense of community in the classroom. First, the Classroom Observation Protocol for Undergraduate STEM (COPUS) [9] is a protocol designed for use in university settings to generate information on the frequency of different instructional strategies occurring in the classroom. This instrument provides information on the impact of the interventions by measuring what the instructor and students are doing at twominute intervals. The COPUS protocol will be used at regular intervals throughout the term in both sections. COPUS results are analyzed by calculating the frequency of each individual code as a percentage of total codes recorded for the class. Results of the COPUS provide detailed data on the impacts of the micro-interventions on classroom activity and participation.

The Classroom Community Scale [7] is used to measure the sense of community in the classroom. The survey consists of 20 items on a 5-point Likert scale, and includes two subscales: connectedness and learning. The "connectedness" sub-scale captures the students' sense of cohesion, community spirit, trust, and interdependence in the classroom. The "learning" subscale captures students' sense of shared educational goals and the educational benefits they feel they gain by interacting with others in the class. Data analysis for the Classroom Community Scale included descriptive statistics comparing pre/post data across groups, revealing trends to inform the second iteration in Fall 2017. We anticipated higher scores on classroom community 
as a result of the interventions, with higher increases anticipated for students who are traditionally underrepresented in engineering, such as women and minorities.

Results from the quantitative measures will inform a diversity sampling of students at the end of each semester for semi-structured interviews based on classroom community scores. We have developed an interview protocol to illustrate and determine the factors that cause changes in the quantitative measures. The interviews will be conducted to examine the experiences and perceptions of those participants enrolled in the control and treatment sections of the Statics course. Each interview will be transcribed and coded for factors contributing to changes in students' sense of community in the course sections. The interview data will be analyzed through three concurrent processes: data reduction, data display, and conclusion drawing/verification [10]. A constant comparative analysis across all interviews will reveal patterns in the factors contributing to changes in students' attitudes and perceptions.

\section{Preliminary Results}

All quantitative measures have been collected and final qualitative data collection will occur in early May 2017, including post-course interviews with a sample of students in each section. Initial results show differences in the way in which the initial micro-interventions, centered around creating community in the classroom, are affecting the instructor and student time in the classroom. For example, Figure 2 shows the results of the COPUS protocol averaged over seven observations across the two sections. The treatment section shows more diversity in activities for both the instructor and students as compared to the control section. For example, lecture was coded as $23 \%$ of all recorded codes for the instructor in the treatment cohort, and $38 \%$ of the recorded codes for the instructor of the control cohort, leading to a reduction in the frequency that students were coded as listening (44\% in the treatment course and $60 \%$ in the control course). This shift allowed for more student participation and social interaction around engineering problems leading to, we hypothesize, a stronger sense of community in the course over time as the microinterventions are sustained.

\section{The Classroom} Community Scale instrument (with a maximum score of 80) showed a moderate increase in community scores in the treatment

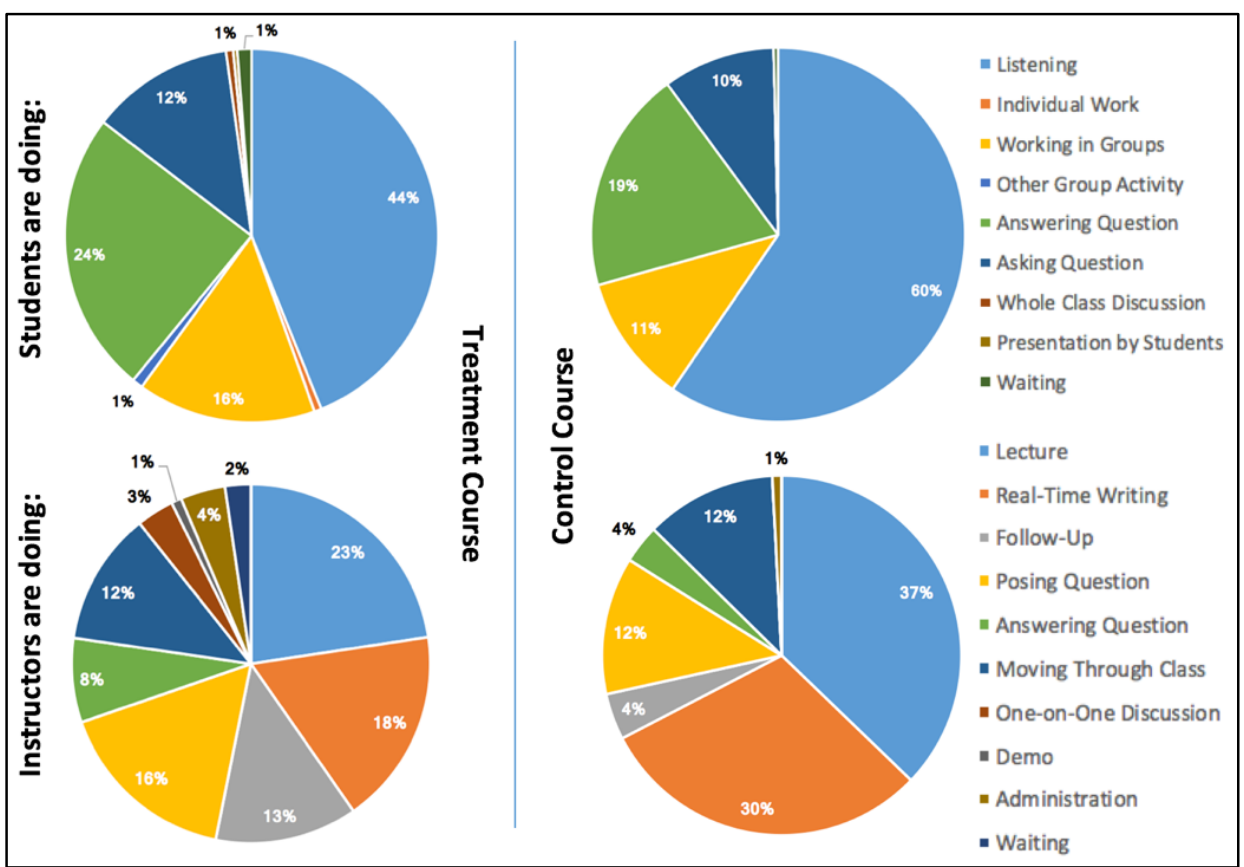

Figure 2. A comparison of COPUS results across the treatment and control groups. Results are the average scores for each section over seven observations. 
group (49 to 53) and no change in the control group (45 to 45). Similar results were found within the sub-scales. It is important to note that these results are skewed toward those students who stayed in the course. The control group experienced a $28 \%$ withdraw rate (as compared to a $13 \%$ rate in the treatment course). It is reasonable to assume those students who chose to withdraw would have scored lower in this measure, further widening the difference between the two groups.

\section{Discussion and Conclusion}

The overall goal of this project is to decrease attrition rates for all students in an introductory engineering course commonly considered a "weed out" course. In the larger project of which this study is a part, we utilize the constructs of engineering identity and self-efficacy as proxies to examine future attrition. In this study, we focus on fine-tuning our instructional interventions to increase students' sense of community. Results from this initial iteration reveal useful differences in the role instructors and students play in the course as well as the impact those changes have on students' sense of community. Over time, we believe an increase in a sense of community among the students will have a positive impact on both their engineering identity and self-efficacy, and thus their continuation as engineering majors, as they continue in their programs.

\section{References}

[1] Blickenstaff, J.C. 2005. "Women and science careers: Leaky pipeline or gender filter?" Gender and Education, 17(4), pp. 369-386.

[2] Suresh, R. 2006/2007. "The relationship between barrier courses and persistence in engineering." Journal of College Student Retention, 8(2) pp. 215-39.

[3] Lumsdaine, M. and Lumsdaine, E. 1995. "Thinking preferences of engineering students: Implications for curriculum restructuring." Journal of Engineering Education, 84(2), pp. 193-204.

[4] Smith, T.Y. 2000. "Science, mathematics, engineering and technology retention database." Research News on Graduate Education, 2(2).

[5] National Science Foundation, National Center for Science and Engineering Statistics. 2011. Women, Minorities, and Persons with Disabilities in Science and Engineering: 2011, National Science Foundation, Arlington, VA.

[6] Seymour, E. and Hewitt, N.M. 1997. Talking about leaving: Why undergraduates leave the sciences, Boulder, CO: Westview Press.

[7] Rovai, A. P. 2002. "Development of an instrument to measure classroom community." The Internet and Higher Education, 5(3), pp. 197-211.

[8] Courter, S. S., Millar, S. B., and Lyons, L. 1998. "From the students' point of view: Experiences in a freshman engineering design course." Journal of engineering education, 87(3), pp. 283-288.

[9] Smith, M. K., Jones, F. H., Gilbert, S. L., and Wieman, C. E. 2013. "The Classroom Observation Protocol for Undergraduate STEM (COPUS): A new instrument to characterize university STEM classroom practices." CBE-Life Sciences Education, 12(4), pp. 618-627.

[10] Miles, M. B., \& Huberman, A. M. 1994. Qualitative data analysis: An expanded sourcebook. Thousand Oaks, CA: Sage Publications. 